\title{
ESTRATEGIAS DE APRENDIZAJE, MOTIVACIÓN Y RENDIMIENTO ACADÉMICO EN ALUMNOS UNIVERSITARIOS
}

\author{
UNDERGRADUATE STUDENTS' LEARNING STRATEGIES, \\ MOTIVATION, AND ACADEMIC PERFORMANCE
}

\author{
J. Reynaldo Martínez* y Ferrán Galán** \\ Universidad de Barcelona
}

\section{RESUMEN}

En el presente trabajo se estima la consistencia interna del MSLQ (autoreporte de estrategias de aprendizaje y motivación) en una muestra de estudiantes universitarios de Pedagogía de la Universidad de Barcelona y la correlación entre las estrategias autorreportadas y la calificación definitiva en una asignatura específica. Participan 182 estudiantes de $1^{\text {er }}$. curso de Pedagogía, la mayoría de sexo femenino (88\%) y con una edad promedio de 19,63 años. Para el área de motivación (alpha $=.65)$, un análisis factorial confirmatorio determina que las subescalas de: valor de la tarea, autoeficacia, orientación extrínseca, ansiedad y control de creencias son consistentes para su constructo. En cuanto a las estrategias de aprendizaje (alpha $=.73$ ), destacan las subescalas de: elaboración, organización, tiempo y ambiente de estudio, y autorregulación. En relación con la calificación definitiva no se evidencia relación significativa entre esta y las creencias del sujeto sobre su motivación y sus estrategias de aprendizaje. El hecho de no hallar relación lineal entre las estrategias autoreportadas por el alumno y su calificación definitiva en la asignatura, puede estar explicado por: (1) deficiencias en la práctica instruccional, (2) deficiencias en la evaluación final de la asignatura (tipo test de selección múltiple), y/o (3) problemas de validez externa del MSLQ en esta muestra de universitarios españoles. Se discuten estas posibles explicaciones.

Palabras clave: motivación, estrategias de aprendizaje, educación superior, validación.

* Licenciado en Pedagogía. Master en Procesos de Aprendizaje. Becario de investigación en el Departamento de Psicología Básica de la Universidad de Barcelona. Doctorando en Psicología por la Universidad de Barcelona. Su principal línea de investigación son los procesos de enseñanza-aprendizaje en la universidad.

** Estudiante del último año de carrera en Psicología. Practicante-colaborador en el Departamento de Psicología Básica de la Universidad de Barcelona.

Este trabajo forma parte del proyecto: Estrategias de enseñanza-aprendizaje en la Universidad. Subvencionado por una beca del Instituto de Cooperación Iberoamericana-ICI, España y apoyo económico de la Vollmer Foundation - Caracas, Venezuela. 


\begin{abstract}
In this study the main purpose was to estimate the internal consistency of the MSLQ (Motivated Strategies for Learning Questionnaire) and to estimate the correlation between the academic performance, cognitive and motivational strategies. Participants were 182 undergarduates ( $88 \%$ female) in a pedagogy program at the University of Barcelona. Data analysis consisted in the use of descriptive statistics, Spearman correlation and factor analysis. The results suggest that in the motivation scale (alpha $=$ .65), intrinsic goal orientation, task value, selfefficacy and control beliefs have strong consistency. In the learning strategies section (alpha $=.73$ ) four subscales are consistent: elaboration, critical thinking, regulational, and time and study enviroment. The subscales showed no significant correlations with final grades and we believe that this lack of correlation is due to: (1) deficiences in teaching practice, (2) deficiences in evaluation performance, (3) a possible lack of cross cultural validity of the MSLQ.
\end{abstract}

Key words: motivation, learning strategies, higher education, validity.

\title{
Introducción
}

A partir del vertiginoso avance de las teorías cognitivas y constructivistas en psicología y educación, se plantea colocar el énfasis en los procesos de adquisición del conocimiento vs. una tradición centrada en resultados o productos. Es así, como surge un conjunto de preceptos teóricos acerca del proceso de enseñanza-aprendizaje, orientados a optimizar la práctica educativa, los cuales asignan un valor importante a las «estrategias de aprendizaje» del alumno y la acción mediadora del docente, colocando el énfasis en los procesos conscientes que emplean alumnos y profesores para enfrentar las tareas que requiere la óptima adquisición del conocimiento.

En esta línea comienzan a destacar una serie de instrumentos y tareas que se orientan a la medición de los procesos internos del alumno (cognitivos y/o motivacionales); entre ellos los estudios de Pintrich y Col., han sido ampliamente reseñados y destacados en los años recientes, abriendo un extenso espacio de investigación entre las variables cognitivas, las motivacionales y los resultados en el rendimiento académico o en el desempeño en la solución de tareas específicas.

En tal sentido, se destaca la correlación positiva entre las estrategias de aprendizaje y las motivacionales, como factor clave en la explicación del buen rendimiento académico (Pintrich and DeGroot, 1990). Con base en estos elementos, este estudio se propuso dos objetivos: a) estimar la consistencia interna del MSLQ (autorreporte de estrategias de aprendizaje y motivación), y b) estimar la correlación entre las estrategias autorreportadas y el rendimiento académico, en un grupo de estudiantes universitarios de pedagogía de la Universidad de Barcelona.

Con este trabajo, se amplía la muestra de estudiantes universitarios que en España, Latinoamérica y USA; han autorreportado sus estrategias de aprendizaje y motivacionales a través del MSLQ (Pintrich, et al., 1988) o versiones castellanas adaptadas del original, como los estudios de Roces, Tourón y González (1995a, b) y los de Castañeda (1997). Cabe destacar que al igual que los autores mencionados, empleamos este cuestionario, ya que en su concepción metodológica se asume un modelo teórico basado en que los efectos de la instrucción son mediados por las estrategias cognitivas y motivacionales del estudiante (Pintrich, 1990); y tal como expresan Roces, Tourón y González (1995b), en este cuestionario de autorreporte, observamos un buen conjunto de items que representan los componentes cognitivo y motivacional de las estrategias de aprendizaje. 


\section{Método}

\section{Muestra}

Participan 182 estudiantes de $1^{\text {er }}$ curso de Pedagogía en la Universidad de Barcelona que inscribieron la asignatura Procesos Psicológicos Básicos, turno de la mañana, en el curso académico 1997/98. Originalmente se obtuvo información de 193 estudiantes, que conformaban el total de inscritos, pero se seleccionaron únicamente los estudiantes que cursaban la asignatura por primera vez y se ubicaban en el $1^{\text {er }}$ año de carrera (182), la mayoría de sexo femenino ( $88 \%$ ) y una edad promedio de 19,63 .

\section{Instrumento}

The Motivated Strategies for Learning Questionnaire (MSLQ) (Pintrich et al., 1988, 1993). Cuestionario de autorreporte que evalúa las orientaciones motivacionales de los estudiantes y el uso de diferentes estrategias de aprendizaje en una asignatura específica. Está basado en una visión cognitiva de la motivación y las estrategias de aprendizaje, por lo tanto se considera al estudiante como un procesador activo de la información mediado por su cognición y sus creencias. Desarrollado entre 1982 y 1986 por investigadores de la Universidad de Michigan. La versión original, incluye 81 items entre las dos partes (motivación y estrategias de aprendizaje), los cuales son respondidos por el estudiante señalando un valor entre 1 y 7 (escala likert) que significa «no me describe en absoluto» (valor 1) hasta «me describe totalmente» (valor 7). Se responde en el aula de clase y toma aprox. $30 \mathrm{~min}$. La escala de motivación incluye 31 items y la de estrategias de aprendizaje 50 items (31 sobre estrategias cognitivas y metacognitivas y 19 concernientes a estrategias de manejo de recursos). Adicionalmente, cabe mencionar que las diferentes escalas se han diseñado de forma modular y pueden ser usadas en su totalidad o por separado, de forma simple, según las necesidades del investigador o instructor (Pintrich et. al, 1993).

La versión utilizada en este estudio se compone de 45 items (25 de la sección motivación y 20 de la sección estrategias de aprendizaje) (ver anexo 1), se descartaron items que carecían de interés para el estudio e items de difícil traducción o no adecuados a las características de la muestra y la asignatura. En los cuadros 1 y 2 se observará la distribución hipotética de los items seleccionados, según la estructura propuesta por Pintrich et al., (1988), la cual empleamos como base para el análisis de la distribución de los items según los datos

\section{CUADRO 1: Escala de motivación.}

\begin{tabular}{|l|l|l|}
\hline COMPONENTES & \multicolumn{1}{|c|}{ SUBESCALAS } & \multicolumn{1}{c|}{ ITEMS } \\
\hline Valor. & Orientación intríseca. & $1,16,22$ \\
& Orientación extrínseca. & $7,11,13,30$ \\
& Valor de la tarea. & $4,10,17,26$ \\
\hline Expectancia. & Control sobre creencias & $2,9,18,25$ \\
& Autoeficacia. & $5,6,12,15,20,21$ \\
\hline Emoción. & Test de ansiedad. & $3,8,14,19$ \\
\hline
\end{tabular}


CUADRO 2: Escala de estrategias de aprendizaje.

\begin{tabular}{|l|l|l|}
\hline \multicolumn{1}{|c|}{ COMPONENTES } & \multicolumn{1}{|c|}{ SUBESCALAS } & \multicolumn{1}{c|}{ ITEMS } \\
\hline Estrategias cognitivas y y & Elaboración. & $92,95,109$ \\
metacognitivas. & Organización. & 60,70 \\
& Pensamiento Crítico. & $66,94,99$ \\
& Autorregulación metacognitiva. & $64,69,72,104$ \\
\hline Manejo de recursos. & Tiempo y ambiente de estudio. & $63,71,93,98,101$ \\
& Regulación del esfuerzo. & $62,102,103$ \\
\hline
\end{tabular}

reportados por esta muestra de estudiantes y como hipótesis estructural que oriente la consistencia del autorreporte con otros grupos.

Adicionalmente, se consideró la calificación definitiva de la asignatura en estudio. Esta consistió en una prueba tipo test de selección múltiple con 50 items, en la cual el número de aciertos determinaba la puntuación definitiva, y se considera como el indicador de desempeño académico, porque así lo interpretan los estudiantes y el sistema educativo, aunque necesariamente no es un indicador de aprendizaje.

\section{Análisis de los datos}

Para el análisis del autorreporte (MSLQ) se realizó análisis factorial confirmatorio entre los items de cada una de las escalas, con el objetivo de determinar cómo se agrupaban los items para cada escala y subescala, basándonos en la estructura hipotética definida por Pintrich et al., (1988) y tomando como referencia el estudio de validación con universitarios españoles de Roces et al., (1995b). Los análisis realizados siguieron las orientaciones metodológicas de Renom Pinsach (1998) referidas al análisis factorial.

\section{Resultados}

\section{Análisis factorial de la Escala motivacional}

Tomando como punto de referencia la estructura hipotética propuesta por Pintrich et al., (1988), se realizó análisis factorial de carácter confirmatorio con los 25 items seleccionados de la escala motivacional (ver tabla 1). Se realizó rotación oblimin y se cumplen los requisitos estadísticos de esfericidad. Se observa que para esta muestra se obtienen seis factores o subescalas, los cuales representan con cierta similitud, cinco de las seis subescalas propuestas por Pintrich et al., (excepto la subescala de orientación intrínseca). Destaca el hecho de que todos los items hipotéticamente ubicados en la subescala de valor, se mantienen juntos en nuestra subescala de valor; mientras que los hipotéticamente ubicados en autoeficacia se mantienen juntos, distribuyéndose en nuestro estudio en las subescalas de autoeficacia y en la de expectativa. En resumen, a partir del análisis factorial, tenemos un total de 24/25 items (con excepción del 2) ubicados con saturación superior a .30 en uno de los factores y un total de 18/25 items (72\%) que se ubican en su hipotética subescala, representan- 
TABLA 1: Análisis factorial de la escala motivacional.

\begin{tabular}{|c|c|c|c|c|c|c|}
\hline Item en forma resumida & Valor & $\begin{array}{c}\text { Auto } \\
\text { eficacia }\end{array}$ & $\begin{array}{l}\text { Ore. } \\
\text { extrin. }\end{array}$ & $\begin{array}{c}\text { Ansie- } \\
\text { dad }\end{array}$ & Control & $\begin{array}{l}\text { Expec- } \\
\text { tativa }\end{array}$ \\
\hline $\begin{array}{l}\text { 26. Me gusta esta asignatura } \\
\text { 17. Interesado por la asignatura } \\
\text { 19. Sensación de angustia } \\
\text { 10. Me importa el contenido } \\
\text { 04. Utilizaré lo aprendido } \\
\text { 01. Temas desafiantes } \\
\text { 18. Con mi esfuerzo entenderé }\end{array}$ & $\begin{array}{r}.696 \\
.595 \\
-.515 \\
.475 \\
.402 \\
.401 \\
.378\end{array}$ & & & & & .311 \\
\hline $\begin{array}{l}\text { 15. Entender temas complejos } \\
\text { 06. Entender lecturas difíciles } \\
\text { 16. Temas que estimulen } \\
\text { 20. Hacer excelentes trabajos } \\
\text { 13. Mejores notas que los demás } \\
\text { 12. Aprender conceptos básicos } \\
\text { 22. Entender a fondo el contenido }\end{array}$ & .290 & $\begin{array}{l}-.824 \\
-.590 \\
-.424 \\
-.389 \\
-.374 \\
-.353 \\
-.296\end{array}$ & -.335 & .428 & -.291 & .346 \\
\hline $\begin{array}{l}\text { 11. Mejorar expedicnete } \\
\text { 07. Importancia a la calificación } \\
\text { 30. Demostrar a otros }\end{array}$ & & & $\begin{array}{l}-.643 \\
-.541 \\
-.382\end{array}$ & & & \\
\hline $\begin{array}{l}\text { 08. Pienso preguntas que no sé } \\
\text { 14. Pienso en consecuencias } \\
\text { 03. Menor rendimiento que otros }\end{array}$ & & & & $\begin{array}{l}.593 \\
.509 \\
.339\end{array}$ & & \\
\hline $\begin{array}{l}\text { 09. Es mi culpa si no aprendo } \\
25 \text {. No entiendo. Poco esfuerzo }\end{array}$ & & & & & $\begin{array}{l}.620 \\
.612\end{array}$ & \\
\hline $\begin{array}{l}\text { 21. Espero un buen desempeño } \\
\text { 05. Recibiré un excelente }\end{array}$ & & -.298 & & & & $\begin{array}{l}.582 \\
.383\end{array}$ \\
\hline Varianza explicada por el factor & 20,2 & 8,2 & 7,0 & 6,8 & 5,5 & 5,0 \\
\hline
\end{tabular}

do cinco de las posibles subescalas definidas por Pintrich et al., excepto los items asignados a la subescala de orientación intrínseca $(1,16$ y 22) que saturan en otros factores, diluyendo dicha subescala. En la tabla del análisis factorial, se agregan las saturaciones superiores a .30 en otro factor.

Como puede observarse, en el primer factor o subescala (valor intrínseco de la tarea), saturan los cuatro items correspondientes a valor según la hipótesis estructural de Pintrich et al., $(4,10,17,26)$, los cuales también se agrupan según los resultados de Roces et al., (1995b). Junto a ellos saturan los items 1, 18 y 19 que se asignan originalmente a otras subescalas; sin embargo para esta muestra se agrupan en conjunto con los items de valor, además de ello, por su contenido son asociados al valor intrínseco de la tarea, en este caso hacia una asignatura específica. Cabe destacar que el item 19 satura negativamente con esta subescala, lo cual indica que a menor valor por la asignatura mayor sensación de angustia y viceversa. 
En el segundo factor se agrupan 4/6 de los items correspondientes a autoeficacia $(6,12$, 15, 20), similar a los resultados de Roces et al., (1995b). Se agregan a este segundo factor los item 16 y 13, relacionados con la estimulación de la curiosidad frente a los contenidos difíciles y la obtención de mejores notas. En este factor se observa una mezcla de items sobre autoeficacia al desempeño como meta intrínseca de aprendizaje y el sólo interés por la calificación, similar a los planteamientos de Pintrich et al., (1988). Pero todos se orientan a la confianza de ser capaz de entender el contenido y obtener una buena calificación, por ello mantenemos la denominación de autoeficacia para esta subescala.

El tercer factor agrupa 3/4 de los items correspondientes a orientación extrínseca $(7,11$, 30), dato que también reportan Roces et al., (1995b). El item 13, hipotéticamente unido a los anteriores, pasa al segundo factor. A este tercer factor lo denominamos orientación extrínseca, similar a Pintrich et al., (1988), por ser items orientados específicamente a la calificación y al demostrar a otros los logros.

El cuarto factor agrupa 3/4 de los items asignados al área de ansiedad (3, 8, 14), similar en Roces et al., (1995b). El item 19 ubicado hipotéticamente en esta subescala satura en la de valor de la tarea. En este factor, los items agrupados son relativos a la ansiedad frente a las dificultades de aprender el contenido y sus consecuencias.

El quinto factor agrupa 2/4 de los items relativos a control sobre las creencias de aprendizaje $(9,25)$, también reportado por Roces et al., y de los dos items restantes de la estructura hipotética $(2,18)$; tenemos que el 2 no satura en ningún factor y el 18 en el primer factor, relativo al esfuerzo para entender el contenido de la asignatura, aspecto que consideramos como un indicador de valor por la asignatura y que difiere de los estudios mencionados. Los items ubicados en este factor son relativos a las creencias de control del desempeño en la asignatura.

En el factor seis se ubican los items 21 y 05 los cuales según la estructura hipotética de Pintrich et al., y similar a los resultados de Roces et al., se consideran en conjunto con los items de autoeficacia; sin embargo, para esta muestra aparecen unidos en un factor que por el contenido de los mismos, denominamos expectativa de rendimiento y que se separa de los específicamente asignados a autoeficacia.

Una vez definida la estructura factorial que más se ajustaba a esta muestra, se determinó que entre estos seis factores existe una correlación importante entre las subescalas de valor, autoeficacia y expectativa (ver tabla 2), ello estaría indicando relaciones entre el valor por

TABLA 2: Correlación entre factores motivacionales.

\begin{tabular}{|c|c|c|c|c|c|c|}
\hline & Valor & Autoeficacia & $\begin{array}{c}\text { Or. } \\
\text { extrínseca }\end{array}$ & Ansiedad & Control & Expectativa \\
\hline 1 & - & & & & & \\
\hline 2 & -.275 & - & & & & \\
\hline 3 & -.153 & .138 & - & & & \\
\hline 4 & .102 & .082 & -.041 & - & & \\
\hline 5 & .067 & -.117 & -.079 & .101 & - & \\
\hline 6 & .263 & -.288 & -.147 & -.076 & .103 & - \\
\hline
\end{tabular}


la asignatura, la autoeficacia y las expectativas de rendimiento académico. Para la escala de motivación se obtiene un alpha de .65 .

\section{Análisis Factorial para la Escala de estrategias de aprendizaje}

Para esta escala se realizó un proceso de análisis similar a el de la escala motivacional, en tal sentido, se consideran los resultados de Roces et al., (1995b) y se realiza un análisis factorial confirmatorio a partir de la estructura hipotética de Pintrich et al., (1988) (ver tabla 3). El análisis factorial efectuado, corrobora la congruencia entre los items de pensamiento crítico, organización y tiempo y ambiente de estudio, que saturan en conjunto. La hipotética escala de autorregulación metacognitiva propuesta por Pintrich et al., (1988), se diluye en otros factores. En resumen, se obtienen cuatro factores o subescalas que generan una distribución de los items, diferente a la propuesta por Pintrich et al. En líneas generales, tenemos que 18/20 de los items seleccionados (con excepción de los items 62 y 72), saturan en algún factor. De ellos, 13/20 (65\%) satura en conjunto con los items propuestos por Pintrich et al., (1988). De las subescalas propuestas: elaboración, tiempo y ambiente de estudio y regulación del esfuerzo, mostraron algún item que no saturó tal como se esperaba $(95,101$ y 62 , respectivamente).

TABLA 3: Análisis factorial para estrategias de aprendizaje.

\begin{tabular}{|c|c|c|c|c|c|}
\hline Item en forma resumida & $\begin{array}{c}\text { Elabora- } \\
\text { ción }\end{array}$ & $\begin{array}{l}\text { Organi- } \\
\text { zación }\end{array}$ & $\begin{array}{l}\text { Tiempo y } \\
\text { ambiente }\end{array}$ & $\begin{array}{l}\text { Autorre- } \\
\text { gulación }\end{array}$ & Asistencia \\
\hline $\begin{array}{l}\text { 99. Pensar en alternativas } \\
\text { 66. Cuestionar la asignatura } \\
\text { 94. Elaborar ideas propias } \\
\text { 109. Aplicar ideas } \\
\text { 92. Relacionar el material } \\
\text { 64. Elaborar preguntas }\end{array}$ & $\begin{array}{l}.828 \\
.746 \\
.569 \\
.550 \\
.473 \\
.429\end{array}$ & .427 & & & \\
\hline $\begin{array}{l}\text { 95. Hacer resúmenes breves } \\
\text { 69. Releer y tratar de entender } \\
\text { 60. Subrayar el material } \\
\text { 70. Encontrar idea principal }\end{array}$ & & $\begin{array}{l}.608 \\
.583 \\
.530 \\
.482\end{array}$ & & & \\
\hline $\begin{array}{l}\text { 98. Ritmo de trabajo semanal } \\
\text { 71. Buen uso del tiempo } \\
\text { 93. Espacio privado de estudio } \\
\text { 63. Lugar para concentrarme }\end{array}$ & & & $\begin{array}{l}.725 \\
.540 \\
.420 \\
.307\end{array}$ & & \\
\hline $\begin{array}{l}\text { 104. Qué no entiendo } \\
\text { 103. Identifico ayuda } \\
\text { 102. Persistir en el material }\end{array}$ & & .403 & & $\begin{array}{l}.482 \\
.480 \\
.449\end{array}$ & -.435 \\
\hline 101. Asistir a clases & & & & & -.514 \\
\hline Varianza explicada / c. factor & 22,4 & 11,3 & 7,4 & 6,6 & 6,0 \\
\hline
\end{tabular}


En el primer factor saturan los items de pensamiento crítico $(66,94,99)$, se agregan $2 / 3$ de elaboración $(92,109)$ y el item 64 agrupado por Pintrich et al., (1988), en una subescala de autorregulación que en esta muestra se diluye en varios factores. En relación a los resultados de Roces et al., (1995b), tenemos que estos datos son similares a la subescala de elaboración, término que mantenemos para este primer factor, por el contenido de los items agrupados, los cuales se refieren a las estrategias de procesamiento, de combinación y de relación entre la información adquirida.

El segundo factor agrupa a los items de organización $(60,70)$ y se agregan el 69 (de autorregulación) y el 95 (de elaboración). A este factor lo denominamos organización, ya que los items aquí contenidos se refieren a estrategias de organización de la información. En relación con los datos de Roces et al., (1995b), hallamos similitud con la subescala de organización que entre otros, agrupa los items 60, 70 y 95 de este estudio.

El tercer factor agrupa 4/5 de los items de tiempo y ambiente de estudio $(63,71,93,98)$ con excepción del 101 (asistencia a clase) que se ubica como item único en un quinto factor. Este tercer factor, conforma una subescala muy similar a la propuesta por Pintrich et al., (1988), con la que coincidimos en su denominación y no se corresponde con ninguna de las subescalas de Roces et al., (1995b). Cabe destacar que dado que la asignatura se estructuraba en secciones teóricas y prácticas, en días y horarios diferentes, la asistencia no era igual para toda la asignatura y ello pudo ser una razón importante para que las respuestas al item 101 no fueran homogéneas y de allí que sature en otro factor. Cabe destacar que se detecta una relación negativa con el item de persistencia con el material de estudio.

El cuarto factor agrupa items de manejo de recursos $(102,103)$ y de autorregulación (104), según la estructura hipotética de Pintrich et al., sin embargo, por su relación de contenidos (determinar qué no entiendo, identificar ayuda y persistencia), definimos a esta subescala: autoregulación, variando la estructura hipotética propuesta. Esta agrupación tampoco se corresponde con ninguna de las subescalas definidas por Roces et al., (1995b).

Una vez definidas las diversas subescalas para estrategias de aprendizaje, hallamos que entre los cinco factores extraídos existe correlación entre el 2 y el 3 , y entre el 3 y el 4 (ver tabla 4). La correlación hallada entre los factores 3 y 4 corrobora el componente de manejo de recursos definido por Pintrich et al., mientras que la relación entre los factores 2 y 3 (organización y tiempo de estudio), puede estar explicada, por el hecho de que las estrategias de organización de la información se consideren como parte del manejo del tiempo y los re-

TABLA 4: Correlación entre factores de estrategias de aprendizaje.

\begin{tabular}{|c|c|c|c|c|c|}
\cline { 2 - 6 } \multicolumn{1}{c|}{} & Elaboración & Organización & $\begin{array}{c}\text { Tiempo y } \\
\text { ambiente }\end{array}$ & $\begin{array}{c}\text { Autorregu- } \\
\text { lación }\end{array}$ & Asistencia \\
\hline 1 & - & & & & \\
\hline 2 & .118 & - & & & \\
\hline 3 & .221 & .357 & - & & \\
\hline 4 & .179 & .106 &. $\mathbf{2 7 6}$ & - & \\
\hline 5 & -.174 & -.233 & -.168 & .021 & - \\
\hline
\end{tabular}


cursos, dejando a las estrategias de pensamiento crítico como otro nivel de estrategias. Para esta escala se obtuvo un alpha de .73.

\section{Estrategias de aprendizaje, motivación y calificación definitiva}

Una vez aplicado el cuestionario de autorreporte y realizado el proceso de análisis factorial de los items, se construyeron las diversas subescalas definidas para la muestra. De esta manera se obtuvo el valor específico para cada una de las subescalas o factores correspondientes a estrategias de aprendizaje y motivación, y se realizó análisis correlacional entre ellas y la calificación definitiva en la asignatura en estudio (ver tabla 5).

\section{TABLA 5: Correlaciones entre estrategias de aprendizaje, motivación y rendimiento académico.}

\begin{tabular}{|c|c|c|c|c|c|c|c|c|c|c|}
\hline Variable & 1 & 2 & 3 & 4 & 5 & 6 & 7 & 8 & 9 & 10 \\
\hline 1. Valor & - & $.40^{*}$ & $.26 *$ & .03 & .18 & $.27 *$ & $.53 *$ & $.26 *$ & $.35 *$ & $.24 *$ \\
\hline 2. Autoeficacia & & - & $.33 *$ & -.06 & .09 & $.46 *$ & $.28 *$ & .21 & .19 & .12 \\
\hline 3. Or. Extrínseca & & & - & .08 & -.04 & .14 & .09 & .14 & .20 & $.31 *$ \\
\hline 4. Ansiedad & & & & - & .23 & -.14 & .13 & -.02 & .04 & .21 \\
\hline 5. Control & & & & & - & .05 & .19 & .06 & .01 & -.03 \\
\hline 6. Expectativa & & & & & & - & .13 & $.28 *$ & .16 & .11 \\
\hline 7. Elaboración & & & & & & & - & .16 & $.32 *$ & .23 \\
\hline 8. Organización & & & & & & & & - & $.39 *$ & .20 \\
\hline 9. Tiempo y ambiente de estudio & & & & & & & & & - & $.27 *$ \\
\hline 10. Autorregulación & & & & & & & & & & - \\
\hline NOTA & .10 & -.02 & .03 & .05 & -.10 & -.06 & .01 & -.01 & .08 & .09 \\
\hline
\end{tabular}

$\mathrm{N}=182 ; \quad * \mathrm{p}<.001$

Se observa un conjunto de correlaciones significativas entre las diversas subescalas (de aprendizaje y motivación); sin embargo, no se halló relación significativa con la calificación definitiva en la asignatura. De las correlaciones entre las subescalas motivacionales y las de estrategias de aprendizaje, destaca la relación significativa entre valor intrínseco de la asignatura y todas las subescalas sobre estrategias de aprendizaje, datos similares a los reportados por Pintrich y De Groot (1990), lo cual implica que a mayor valor por la asignatura mayor disposición para emplear estrategias de aprendizaje y viceversa. Sin embargo, el hecho de no hallar correlación significativa con la calificación final en la asignatura es un dato sumamente curioso que no se corresponde con lo destacado en la bibliografía y que genera una llamada de atención a los procedimientos instruccionales y al sistema de evaluación, como posibles hipótesis que obstruyen la mediación del rendimiento académico a través de los procesos internos del alumno. 


\section{Discusión y conclusiones}

Con base en el análisis realizado al MSLQ, podemos señalar que para esta muestra de universitarios españoles, se obtiene una estructura consistente con la propuesta por Pintrich et al., (1988). En tal sentido, se detectó una buena congruencia interna en relación con las subescalas del área de motivación (72\%) y aceptable para el área de estrategias de aprendizaje $(65 \%)$. Asimismo, cabe destacar que se halló una alta correspondencia con los resultados de Roces et al., (1995b).

Para el área de motivación, se obtienen cinco subescalas bien definidas y que corresponden con la estructura hipotética de Pintrich et al., y los datos reportados por Roces et al., no se obtuvo una subescala específica para orientación intrínseca, sino una redistribución de sus items en unión con los items referidos al valor de la tarea.

Para el área de estrategias de aprendizaje, se muestra una falta de consistencia con el modelo de Pintrich et al., en esta muestra, sólo se logran reproducir las subescalas de elaboración, organización y tiempo y ambiente de estudio. Creemos que esta falta de correspondencia se debe a que las estrategias de manejo de recursos y de autorregulación metacognitiva no están bien definidas en la estructura hipotética de Pintrich et al., (1988) y que además son estrategias difíciles de separar del componente cognitivo, por ello observamos como los diversos items correspondientes a estos factores se distribuyen en otras subescalas. Tampoco obtenemos una alta congruencia con las subescalas definidas por Roces et al., (1995b), sino sólo en dos de los seis factores propuestos.

En resumen, podemos señalar que el MSLQ muestra una buena congruencia interna para el área de motivación y una estructura débil para el área de estrategias de aprendizaje, lo cual indica que es un instrumento óptimo en la medición de las estrategias de aprendizaje que emplean los alumnos universitarios, tal como destacan Pintrich et al., (1993, 1994a, 1994b) en USA. Roces, González y Tourón (1997); Roces, Tourón y González (1995b) en España y Castañeda (1997) en México. Sin embargo, en todos estos estudios los datos de estrategias de aprendizaje varían en su estructura y quizás ello responda a una explicación contextual o a una falta de definición clara de los constructos que conforman esta escala.

En cuanto la calificación final no se evidencia relación significativa entre las subescalas que miden las creencias del sujeto sobre su actuación estratégica, su motivación y el resultado final en la asignatura. En este sentido, creemos que esta no relación puede estar explicada por:

1. Deficiencias en la práctica instruccional, divorciada de los preceptos teóricos constructivistas y centrada en la acción expositiva del profesor y la actitud pasiva -«repetitiva»- del alumno, lo cual no permite que las estrategias del alumno sean mediadoras de su rendimiento académico.

2. Deficiencias en la evaluación, centrada en resultados y no en procesos, determinada por una prueba tipo test de selección múltiple, para decidir la promoción o no del alumno, práctica divorciada de la propuesta teórica del enfoque del procesamiento de la información y de la epistemología constructivista. Con este tipo de evaluación es poco probable que se puedan hallar relaciones con las estrategias de aprendizaje, a menos que nos centremos sólo en las prácticas memorísticas y expositivas. En palabras de Roces et al., (1997), «los alumnos pueden creer que son capaces de apren- 
der, y sin embargo no esperar (y no obtener) un buen rendimiento porque piensan que las exigencias del profesor o el sistema de evaluación no sean los adecuados».

3. Probablemente, otra hipótesis es que el MSLQ carezca de validez externa para esta muestra, sin embargo, su alta consistencia interna y resultados de otras investigaciones con estudiantes españoles (ver Roces et al.), nos hacen dudar de esta explicación a la no-relación entre las estrategias de aprendizaje del alumno y su rendimiento académico final.

En resumen, tenemos un conjunto de resultados sumamente interesantes que deben generar una revisión de los procesos instruccionales, mecanismos de evaluación del rendimiento en la Universidad y afinar los instrumentos de evaluación psicológica en esta población. Para nosotros no cabe pensar (teóricamente hablando) la existencia de no relación entre las habilidades que autorreporta el sujeto, tan congruentemente, y su rendimiento. Por lo tanto, consideramos que el MSLQ es un buen instrumento de medida y que la práctica instruccional en la enseñanza universitaria no está valorando las estrategias del alumno y su motivación, los cuales deberían ser factores claves en la formación académica y por lo tanto, justamente valorados y promovidos.

\section{Referencias}

Castañeda, S. (1997): «Cognitive, affective-motivational and social variables in the teaching of science and techniques». Ponencia presentada en: Congreso Regional de Psicología para profesionales en América, México, 27 de julio al 2 de agosto.

PINTRICH, P. (1990): «Implications of psychological research on student learning and college teaching for teacher education». In W. R. Houston (Ed.), Handbook of research on teacher education (pp. 826-856): New York: Macmillan Publishing.

PINTRICH, P., et al. (1994a): «Classroom and individual differences in early adolescents' motivation and self-regulated learning». Journal of Early Adolescence, 14, (2), 139-161.

Pintrich, P., Anderman, E. and Klobucar, Ch. (1994b): «Intraindividual differences in motivation and cognition in students with and without learning disabilities». Journal of Learning Disabilities, $27,(6), 360-370$.

PINTRICH, P. and DeGroot, E. (1990): «Motivational and self-regulated learning components of classroom academic performance». Journal of Educational Psychology, 82, (1), 33-40.

Pintrich, P., McKeachi, W., Smith, D., Doljanac, R., Lin, Y., Naveh-Benjamin, M., Crooks, T. and KARABENICK, S. (1988): Motivated strategies for learning questionnaire. The University of Michigan (NCRIPTAL).

Pintrich, P., Smith, D., García, T. y Mckeachie, W. (1993): «Reliability and predictive validity of the motivated strategies for learning questionnaire (MSLQ)». Educational and Psychological Measurement, 53, 801-813.

Renom Pinsach, J. (1998): Tratamiento informatizado de datos. Barcelona: Masson.

Roces, C., GonzÁlez, M. C. y Tourón, J. (1997): «Expectativas de aprendizaje y de rendimiento de los alumnos universitarios». Revista de Psicología de la Educación, (22), 99-123.

Roces, C., Tourón, J. y GonZÁLez, M. C. (1995a): «Motivación, estrategias de aprendizaje y rendimiento de los alumnos universitarios». Bordón, 47, (1), 107-120.

Roces, C., Tourón, J. y GonZÁLEZ, M. C. (1995b): «Validación preliminar del CEAM II» (cuestionario de estrategias de aprendizaje y motivación II). Psicológica, 16, 347-366. 


\section{ANEXO 1}

\section{Parte A. Motivación}

Las siguientes cuestiones indagan respecto a tu motivación y actitudes en esta asignatura. Usa la escala de abajo para contestar las preguntas. Si piensas que un enunciado te describe totalmente, marca el 7; si el enunciado no te describe en absoluto, marca el 1. Si el enunciado te describe mas o menos, escoge el número entre el 1 y el 7 que mejor te describa.

1. No me describe en absoluto.

2. Me describe un poco.

3. Me describe moderadamente.

4. No estoy seguro(a).

5. Me describe suficientemente.

6. Me describe mucho.

7. Me describe totalmente.

1. En una asignatura como esta prefiero que el contenido de la clase sea desafiante, de tal modo que pueda aprender cosas nuevas.

2. Si estudio en la forma apropiada podré aprender el material o contenido de esta asignatura.

3. Cuando presento una tarea, examen u otra actividad en esta asignatura pienso que mi desempeño es deficiente comparado con el de mis compañeros.

4. Pienso que seré capaz de usar lo que aprenda en esta asignatura para otros cursos.

5. Creo que recibiré una excelente calificación en esta asignatura.

6. Estoy seguro de que puedo entender las lecturas más difíciles de esta asignatura.

7. Conseguir una buena calificación en esta asignatura es la cosa más satisfactoria para mi hasta ahora.

8. Cuando presento una prueba o tarea en esta asignatura, pienso en los items o preguntas de la misma que no he podido contestar.

9. Es mi culpa si no aprendo el material o contenido de esta asignatura.

10. Es importante para mí aprender el material de esta asignatura.

11. La cosa más importante para mi ahora es mejorar mi promedio general, por lo que mi principal interés en esta asignatura es conseguir una buena calificación.

12. Confío en que puedo aprender los conceptos básicos enseñados en esta asignatura.

13. Si quiero, puedo conseguir las mejores calificaciones en esta asignatura y superar a mis compañeros.

14. Cuando presento una tarea, examen o actividad en esta asignatura pienso en las consecuencias de un fracaso. 
15. Confío en que puedo entender el material más complejo presentado por el profesor en este asignatura.

16. En una asignatura como esta, prefiero que el material o contenido aliente mi curiosidad, aun si es difícil de aprender.

17. Estoy muy interesado en el contenido de este asignatura.

18. Si me esfuerzo lo suficiente, entenderé el contenido de la asignatura.

19. En este asignatura, experimento una sensación desagradable como de «angustia».

20. Confío en que puedo hacer un excelente trabajo respecto a las tareas y exámenes en este asignatura.

21. Espero que mi desempeño en esta asignatura sea bueno.

22. La cosa más satisfactoria para mí en esta asignatura es tratar de entender el contenido tan completamente como sea posible.

23. Pienso que me es útil aprender el contenido de este asignatura.

24. Cuando tengo la oportunidad escojo las tareas de la asignatura en las cuales pueda aprender, aun si ello no me garantiza una buena calificación.

25. Si no entiendo el contenido de la asignatura es porque no me esfuerzo lo necesario.

26. Me gusta esta asignatura.

27. Entender esta asignatura es muy importante para mí.

28. Siento angustia cuando presento un examen o tarea en esta asignatura.

29. Estoy seguro de que puedo dominar las habilidades que se enseñan en esta asignatura.

30. Quiero desempeñarme bien en esta asignatura porque es importante para mi, demostrar mi habilidad a mi familia, amigos, jefe u otros.

31. Considerando la dificultad de esta asignatura, el profesor y mis habilidades; pienso que saldré bien en el resultado final.

\section{Parte B. Estrategias de Aprendizaje}

Los siguientes enunciados investigan tres estrategias de aprendizaje y habilidades de estudio para esta asignatura. Aquí tampoco hay respuestas correctas ni incorrectas. Contesta estos enunciados acerca de cómo estudias para esta asignatura tan fidedignamente como te sea posible. Usa la escala anterior. (1) Si piensas que el enunciado no te describe en absoluto y hasta (7) si te describe completamente.

60. Cuando estudio las lecturas para esta asignatura subrayo el material para ayudarme a organizar mis pensamientos.

61. Durante la asignatura con frecuencia se me escapan puntos importantes, porque estoy pensando en otras cosas.

62. Cuando estudio para esta asignatura, con frecuencia trato de explicar lo estudiado a un compañero o amigo.

63. Usualmente estudio en un lugar donde pueda concentrarme. 
64. Cuando leo para esta asignatura, elaboro preguntas para ayudarme a enfocar mi lectura.

65. Frecuentemente me siento tan perezoso (a) o aburrido (a) cuando estudio para esta asignatura que abandono el estudio antes de finalizar lo que planeaba hacer.

66. Frecuentemente me cuestiono cosas que he oído o leído en esta asignatura para decidir si las encuentro convincentes.

67. Cuando estudio para esta asignatura, practico repitiendo el material para mí mismo una y otra vez.

68. Aun si tengo problemas para aprender el material de esta asignatura, trato de hacerlo solo sin la ayuda de nadie.

69. Cuando estoy confundido acerca de algo que estoy leyendo para esta asignatura, vuelvo a leerlo y trato de entenderlo.

70. Cuando estudio para esta asignatura me baso en las lecturas y mis apuntes y trato de encontrar las ideas más importantes.

71. Uso bien mi tiempo de estudio para esta asignatura.

72. Si las lecturas son difíciles de entender, cambio la forma de leer el material.

73. Trato de trabajar con otros estudiantes de esta asignatura para completar las tareas asignadas.

74. Cuando estudio para esta asignatura, leo las notas tomadas en clase y las lecturas una y otra vez.

75. Cuando una teoría, interpretación o conclusión se presenta en esta asignatura o en las lecturas asignadas, trato de decidir si hay una buena evidencia que la apoye.

76. Trabajo duro para salir bien en esta asignatura, aun si no me gusta lo que estamos haciendo.

77. Hago diagramas, gráficas o tablas simples para ayudarme a organizar el material de la asignatura.

78. Cuando estudio para esta asignatura con frecuencia dedico un tiempo para discutir el material con un grupo de estudiantes de la clase.

79. Tomo el material de la asignatura como un punto de arranque y trato de desarrollar mis propias ideas acerca de él.

80. Se me hace difícil sujetarme a un horario de estudio.

81. Cuando estudio para esta asignatura reúno la información de diferentes fuentes como lecturas, discusiones y notas.

82. Antes de estudiar a profundidad el nuevo material de la asignatura, frecuentemente lo reviso para ver cómo está organizado.

83. Yo mismo me hago preguntas para asegurarme que entiendo el material que he estado estudiando en esta asignatura.

84. Trato de cambiar la forma en que estudio a fin de ajustarla a los requerimientos de la asignatura y al estilo de enseñanza del profesor.

85. Frecuentemente me doy cuenta que he estado leyendo para esta asignatura pero no he comprendido bien las lecturas. 
86. Pregunto al profesor para clarificar conceptos que no entiendo bien.

87. Memorizo palabras claves para recordar conceptos importantes en esta asignatura.

88. Cuando el material y/o las tareas son difíciles, los abandono y sólo estudio las partes fáciles.

89. Trato de pensar sobre un tópico para decidir qué se supone que debo aprender sobre él, más que solamente leerlo y aprenderlo de memoria.

90. Trato de relacionar las ideas de esta asignatura con otras.

91. Cuando estudio para esta asignatura, voy a mis apuntes y subrayo los conceptos importantes.

92. Cuando leo para esta asignatura trato de relacionar el material con el que ya conozco.

93. Tengo un espacio privado para estudiar.

94. Trato de elaborar mis propias ideas acerca de lo que estoy aprendiendo en esta asignatura.

95. Cuando estudio para esta asignatura escribo resúmenes breves de las principales ideas de las lecturas y de mis apuntes.

96. Cuando no puedo entender la asignatura pido a otro estudiante de la clase que me ayude.

97. Trato de entender el material de esta asignatura para hacer conexiones entre las lecturas y los conceptos estudiados.

98. Me aseguro de mantener un ritmo continuo semanal de trabajo en las lecturas y tareas para este asignatura.

99. Siempre que leo o escucho una afirmación o conclusión en esta asignatura, pienso acerca de posibles alternativas.

100. Hago listas de puntos importantes para esta asignatura y las memorizo.

101. Asisto a esta asignatura regularmente.

102. Aun cuando el contenido de esta asignatura es (o fuera) monótono, pesado y nada interesante, persisto (o persistiría) en trabajar sobre él hasta finalizarlo.

103. Trato de identificar en esta asignatura a los estudiantes a los que puedo pedir ayuda si es necesario.

104. Cuando estudio para esta asignatura trato de determinar qué conceptos no entiendo bien.

105. Frecuentemente me percato de que no dedico mucho tiempo a esta asignatura debido a otras actividades.

106. Cuando estudio para esta asignatura establezco mis propios objetivos, para organizar mis actividades en cada período de estudio.

107. Cuando tomo notas que me confunden o no entiendo, las señalo para releerlas más tarde y tratar de entenderlas. 
108. Raramente encuentro tiempo para revisar mis notas o leer sobre esta asignatura, antes de las clases.

109. Trato de aplicar las ideas de las lecturas de esta asignatura en otras actividades, tales como: exposiciones y discusiones.

Fecha de recepción: 18-10-99

Fecha de revisión: 5-11-99

Fecha de aceptación: 11-4-00 